\title{
Catalytic Coupling of Haloolefins with Anilides
}

Vladimir G. Zaitsev and Olafs Daugulis*

\section{SUPPORTING INFORMATION}

S-1 


\section{Experimental Section}

General considerations. The coupling reactions were performed without special precautions in 1-dram screw-cap vials. Flash chromatography was performed on $60 \AA$ silica gel (Sorbent Technologies). GC analyses were performed on a Shimadzu CG-2010 chromatograph equipped with a Restek column (Rtx ${ }^{\circledR}-5,15 \mathrm{~m}, 0.25 \mathrm{~mm}$ ID). The ${ }^{1} \mathrm{H}$ and ${ }^{13} \mathrm{C}$ NMR spectra were recorded on a GE QE-300 spectrometer using residual solvent peak as a reference. Melting points were measured on a Mel-Temp apparatus and are uncorrected. Elemental analyses were performed by Atlantic Microlab Inc. of Norcross, GA. IR-spectra were obtained using a ThermoNicolet Avatar 370 FT-IR instrument. Mass spectra were obtained on a Finnigan Trace GC/MS instrument.

Materials. DMF (Acros), 28-30\% ammonium hydroxide (Merck), 35\% $\mathrm{DCl}$ in $\mathrm{D}_{2} \mathrm{O}$ (Aldrich, 99\% D), 1.6M MeLi in ether (Acros), 1.7M t-BuLi in pentane (Aldrich) and palladium chloride (J\&J Materials) were used as obtained. Silver triflate is available from Strem. Acetanilide, 4-methylacetanilide, 4-bromo-3-methoxyaniline, 2-bromo-4methylaniline are available from Lancaster. The following starting materials were prepared according to literature procedures: methyl cis-3-chloropropenoate, ${ }^{1}$ methyl trans-3-chloropropenoate, ${ }^{1}$ methyl cis-3-bromopropenoate, ${ }^{2}$ methyl trans-3bromopropenoate, ${ }^{2}$ methyl cis-3-iodopropenoate, ${ }^{3}$ cis-1-phenyl-3-bromo-2-propenone, ${ }^{4}$ $\mathrm{N}$-pivaloylaniline, ${ }^{5} \quad 3$-chloro-N-pivaloylaniline, ${ }^{6}$ 3-methoxyacetanilide, ${ }^{7}{ }^{2} \mathrm{~N}$ methylacetanilide, ${ }^{8}$ 3-hydroxy-N-pivaloylaniline, ${ }^{9} \quad 3$-methoxy-N-metylacetanilide, ${ }^{10}$ 2bromo-N(BOC)-4-methylaniline, ${ }^{11}$ 2,6-dideuterio-4-methylaniline hydrochloride. ${ }^{12}$

4-Bromo-3-methoxy-N-pivaloylaniline. To a solution of 4-bromo-3-methoxyaniline $(0.20 \mathrm{~g}, 1.0 \mathrm{mmol})$ and triethylamine $(0.15 \mathrm{~mL}, 1.2 \mathrm{mmol})$ in dry dichloromethane $(20$ $\mathrm{mL})$ was added pivaloyl chloride $(0.16 \mathrm{~mL}, 1.3 \mathrm{mmol})$ and the mixture was stirred at RT for $10 \mathrm{~min}$. The mixture was washed with water $(2 \times 10 \mathrm{~mL})$ and solvent was evaporated in vacuo to give $0.26 \mathrm{~g}(90 \%)$ of a white solid, $\mathrm{mp} 146-147{ }^{\circ} \mathrm{C}\left(\mathrm{CH}_{2} \mathrm{Cl}_{2}\right.$-pentane $) .{ }^{1} \mathrm{H}$ $\operatorname{NMR}\left(\mathrm{CDCl}_{3}\right) \delta 1.32(\mathrm{~s}, 9 \mathrm{H}) ; 3.90(\mathrm{~s}, 3 \mathrm{H}) ; 6.70(\mathrm{dd}, 1 \mathrm{H} ; J=2.1,8.1 \mathrm{~Hz}, \mathrm{CH}=) ; 7.36(\mathrm{br}$ $\mathrm{s}, 1 \mathrm{H}) ; 7.42(\mathrm{~d}, 1 \mathrm{H} ; J=8.1 \mathrm{~Hz}) ; 7.66(\mathrm{~d}, 1 \mathrm{H} ; J=2.1 \mathrm{~Hz}) .{ }^{13} \mathrm{C} \mathrm{NMR}\left(\mathrm{CDCl}_{3}\right) \delta 27.5$; $39.7 ; 56.1 ; 104.3 ; 105.6 ; 112.6 ; 132.7 ; 138.7 ; 156.0 ; 176.8$. Found: $\%$ C 50.85, \%H 5.69, $\% \mathrm{~N} 5.03 . \mathrm{C}_{12} \mathrm{H}_{16} \mathrm{NO}_{2}$. Calculated: $\% \mathrm{C} 50.37, \% \mathrm{H} \mathrm{5.64,} \mathrm{\% N} 4.89$.

Analysis of relative reactivity of haloacrylates with 1. To a solution of palladated complex $1(0.2 \mathrm{~mL}$ of a $0.1 \mathrm{M}$ solution in DMF, as a monomer) was added the respective haloester $(0.2 \mathrm{mmol})$. The mixtures were kept at $80{ }^{\circ} \mathrm{C}$ and periodically analyzed by $\mathrm{GC}$ for accumulation of the product. After $1 \mathrm{hr}$ the following conversions to 2 were observed: methyl cis-2-chloropropenoate, $16 \%$ methyl trans-2-chloropropenoate, $42 \%$ methyl cis-2-iodopropenoate, $51 \%$ methyl cis-2-bromopropenoate, $78 \%$ methyl trans-2-bromopropenoate, $>99 \%$

General procedure for the coupling of methyl trans-3-bromopropenoate with anilides. The solution of anilide $(1.0 \mathrm{mmol})$ in DMF $(1 \mathrm{~mL})$ was mixed with a solution of AgOTf $(282 \mathrm{mg}, 1.1 \mathrm{mmol})$ in $\operatorname{DMF}(0.5 \mathrm{~mL})$ in a 1-dram vial. To this mixture was 
added a solution of $\mathrm{PdCl}_{2}(8.9 \mathrm{mg}, 0.05 \mathrm{mmol})$ in $\mathrm{DMF}(0.25 \mathrm{~mL})$ and neat methyl trans3-bromopropenoate $(110 \mu \mathrm{L}, 1.0 \mathrm{mmol})$. The reaction mixture was heated for $0.5-2 \mathrm{~h}$ at 90-110 ${ }^{\circ} \mathrm{C}$ (for exact conditions, see below). The conversion was monitored by GC. During this time a dark precipitate was formed and the supernatant became clear dark red. The reactions were stopped when no more precipitate was formed. The reaction mixture was diluted with toluene $(30 \mathrm{~mL})$, solution was decanted and the precipitate was washed with toluene $(2 \times 5 \mathrm{~mL})$. Combined organic solutions were washed with water $(10$ $\mathrm{mL})$, brine $(10 \mathrm{~mL})$ and evaporated under reduced pressure. Products were isolated by flash chromatography on silica gel.<smiles>COC(=O)/C=C/c1ccccc1NC(C)=O</smiles>

$90{ }^{\circ} \mathrm{C}$. Purification by flash chromatography $\left(5: 1 \mathrm{CH}_{2} \mathrm{Cl}_{2} / \mathrm{EtOAc}\right)$ afforded $122 \mathrm{mg}(56 \%)$ of the product as a white solid, $\mathrm{mp} 137-138{ }^{\circ} \mathrm{C}\left(\mathrm{CH}_{2} \mathrm{Cl}_{2} /\right.$ hexanes); $\mathrm{R}_{\mathrm{f}}=0.3$ (5:1 $\left.\mathrm{CH}_{2} \mathrm{Cl}_{2} / \mathrm{EtOAc}\right) ;{ }^{1} \mathrm{H}$ NMR $\left(\mathrm{CDCl}_{3}\right) \delta 2.18(\mathrm{~s}, 3 \mathrm{H}), 3.76(\mathrm{~s}, 3 \mathrm{H}) ; 6.35(\mathrm{~d}, 1 \mathrm{H} ; J=15.6 \mathrm{~Hz}$ $\mathrm{CH}=) ; 7.16(\mathrm{t}, 1 \mathrm{H} ; J=7.2 \mathrm{~Hz}) ; 7.33(\mathrm{t}, 1 \mathrm{H} ; J=7.2 \mathrm{~Hz}) ; 7.51(\mathrm{~d}, 1 \mathrm{H} ;=7.5 \mathrm{~Hz}) ; 7.62(\mathrm{~d}$, $1 \mathrm{H} ; J=7.5 \mathrm{~Hz}) ; 7.78(\mathrm{~d}, J=15.6 \mathrm{~Hz}, 1 \mathrm{H}, \mathrm{CH}=) ; 7.79(\mathrm{br} \mathrm{s}, 1 \mathrm{H}) .{ }^{13} \mathrm{C} \mathrm{NMR}\left(\mathrm{CDCl}_{3}\right) \delta$ $23.3,51.8,119.7 ; 125.5 ; 125.9 ; 126.9 ; 127.8 ; 130.7 ; 135.9 ; 139.7 ; 167.2 ; 169.1 ;$ FT-IR $\left(\mathrm{cm}^{-1}\right) 1659$, 1716. Found: $\% \mathrm{C} 65.26, \% \mathrm{H}$ 5.81, \%N 6.27. $\mathrm{C}_{12} \mathrm{H}_{13} \mathrm{NO}_{3}$. Calculated: $\% \mathrm{C}$ $65.74, \% \mathrm{H} 5.98, \% \mathrm{~N} 6.31$.<smiles>COC(=O)/C=C/c1ccccc1NC(=O)C(C)(C)C</smiles>

Methyl (E)-3-(2-pivaloylaminophenyl)propenoate. A 50\% excess of both methyl trans-3-bromopropenoate $(165 \mu \mathrm{L}, 1.5 \mathrm{mmol})$ and silver triflate (385 mg $1.5 \mathrm{mmol}$ ) was used in reaction. Conditions: $1 \mathrm{~h}$ at $90{ }^{\circ} \mathrm{C}$. Purification by flash chromatography $\left(10: 1 \mathrm{CH}_{2} \mathrm{Cl}_{2} / \mathrm{EtOAc}\right)$ afforded $221 \mathrm{mg}(85 \%)$ of the product as a white solid, mp 99-100 ${ }^{\circ} \mathrm{C}$ (pentane); $\mathrm{R}_{\mathrm{f}}=0.45\left(10: 1 \mathrm{CH}_{2} \mathrm{Cl}_{2} / \mathrm{EtOAc}\right) .{ }^{1} \mathrm{H}$ NMR $\left(\mathrm{CDCl}_{3}\right) \delta 1.36$ (s, 9H), 3.79 (s, 3H); 6.38 (d, 1H; $J=14.4 \mathrm{~Hz}, \mathrm{CH}=) ; 7.20$ (t, $1 \mathrm{H} ; J=7.5 \mathrm{~Hz}) ; 7.40$ (dt, $1 \mathrm{H} ; J=1.2$ and $7.5 \mathrm{~Hz}) ; 7.42(\mathrm{br} \mathrm{s}, 1 \mathrm{H}) ; 7.53(\mathrm{dd}, 1 \mathrm{H} ; J=1.2$ and $7.5 \mathrm{~Hz}) ; 7.70(\mathrm{~d}, 1 \mathrm{H} ; J$ $=7.5 \mathrm{~Hz}) ; 7.75(\mathrm{~d}, 1 \mathrm{H} ; J=14.4 \mathrm{~Hz}, \mathrm{CH}=) ;{ }^{13} \mathrm{C}$ NMR $\left(\mathrm{CDCl}_{3}\right) \delta 27.6 ; 39.7 ; 51.8,120.2$; $125.2 ; 125.8 ; 127.2 ; 128.0 ; 130.7 ; 136.0 ; 139.5 ; 167.0 ; 177.0 ;$ FT-IR $\left(\mathrm{cm}^{-1}\right) 1652,1713$. Found: $\% \mathrm{C} 68.89, \% \mathrm{H} 7.36, \% \mathrm{~N}$ 5.32. $\mathrm{C}_{15} \mathrm{H}_{19} \mathrm{NO}_{3}$. Calculated: $\% \mathrm{C} 68.94, \% \mathrm{H} 7.33, \% \mathrm{~N}$ 5.32 . 
<smiles>COC(=O)/C=C/c1cc(C)ccc1NC(C)=O</smiles>

Methyl (E)-3-(2-acetylamino-5-methylphenyl)propenoate (2). A $50 \%$ excess (225 mg, $1.5 \mathrm{mmol})$ of anilide in DMF $(2.0 \mathrm{~mL})$ was used. Conditions: $2 \mathrm{hr}$ at $80{ }^{0} \mathrm{C}$. Flash chromatography $\left(10: 1 \mathrm{CH}_{2} \mathrm{Cl}_{2} / \mathrm{EtOAc}\right)$ afforded $186 \mathrm{mg}(80 \%)$ of the product as a yellow solid, mp $173-174{ }^{\circ} \mathrm{C} ; \mathrm{R}_{\mathrm{f}}=0.3\left(5: 1 \mathrm{CH}_{2} \mathrm{Cl}_{2} /\right.$ EtOAc $) ;{ }^{1} \mathrm{H}$ NMR $\left(\mathrm{CDCl}_{3}\right) \delta 2.22(\mathrm{~s}, 3 \mathrm{H}), 2.33(\mathrm{~s}, 3 \mathrm{H}) ; 3.80(\mathrm{~s}, 3 \mathrm{H}) ; 6.38(\mathrm{~d}, 16.5 \mathrm{~Hz}, 1 \mathrm{H}, \mathrm{CH}=) ; 7.11(\mathrm{~d}$, $8.1 \mathrm{~Hz}, 1 \mathrm{H}) ; 7.20$ (d, $8.1 \mathrm{~Hz}, 1 \mathrm{H}) ; 7.35$ (br s, 1H); 7.55 (d, $8.4 \mathrm{~Hz}, 1 \mathrm{H}) ; 7.78$ (d, $16.5 \mathrm{~Hz}$, $1 \mathrm{H}, \mathrm{CH}=) ;{ }^{13} \mathrm{C} \mathrm{NMR}\left(\mathrm{CDCl}_{3}\right) \delta 20.9 ; 23.7 ; 51.7 ; 119.1 ; 125.9 ; 127.1 ; 128.0 ; 131.4$; $133.5 ; 135.7 ; 139.9 ; 167.3 ; 169.4$; FT-IR $\left(\mathrm{cm}^{-1}\right) 1653,1716$. Found: \%C 67.21, \%H 6.50, $\% \mathrm{~N}$ 5.84. $\mathrm{C}_{13} \mathrm{H}_{15} \mathrm{NO}_{3}$. Calculated: $\% \mathrm{C} 66.94, \% \mathrm{H} 6.48, \% \mathrm{~N} 6.00$. Silver bromide (193 $\mathrm{mg}, 1.0 \mathrm{mmol}$ ) was isolated after drying of the precipitate. It dissolved in $28-30 \%$ aqueous ammonia $(50 \mathrm{~mL})$ leaving no residue.<smiles>COC(=O)/C=C/c1ccc(Cl)cc1NC(=O)C(C)(C)C</smiles>

Methyl (E)-3-(4-chloro-2-pivaloylaminophenyl)propenoate. Conditions: $1.5 \mathrm{hr}$ at $110{ }^{\circ} \mathrm{C}$. Analysis of the crude reaction mixture by GC revealed presence of the coupling product and unreacted anilide. No side products resulting from anilide were observed. Flash chromatography $\left(20: 1 \quad \mathrm{CH}_{2} \mathrm{Cl}_{2} /\right.$ EtOAc) afforded $97 \mathrm{mg}$ (33\%) of the product as a white solid, mp $132-133{ }^{\circ} \mathrm{C}\left(\mathrm{CH}_{2} \mathrm{Cl}_{2} /\right.$ hexanes $) ; \mathrm{R}_{\mathrm{f}}=0.6(10: 1$ $\left.\mathrm{CH}_{2} \mathrm{Cl}_{2} / \mathrm{EtOAc}\right) ;{ }^{1} \mathrm{H}$ NMR $\left(\mathrm{CDCl}_{3}\right) \delta 1.32(\mathrm{~s}, 9 \mathrm{H}) ; 3.76(\mathrm{~s}, 3 \mathrm{H}) ; 6.30(\mathrm{~d}, 16.2 \mathrm{~Hz}, 1 \mathrm{H}$, $\mathrm{CH}=) ; 7.12(\mathrm{~d}, 1 \mathrm{H} ; J=8.1 \mathrm{~Hz}) ; 7.39(\mathrm{~d}, 1 \mathrm{H} ; J=8.1 \mathrm{~Hz}) ; 7.58($ br s, $1 \mathrm{H}) ; 7.61(\mathrm{~d}, 1 \mathrm{H} ; J=$ $16.2 \mathrm{~Hz}, \mathrm{CH}=) .{ }^{13} \mathrm{C} \mathrm{NMR}\left(\mathrm{CDCl}_{3}\right) \delta 27.4 ; 39.7 ; 51.8 ; 120.3 ; 125.0 ; 125.9 ; 126.2 ; 128.0$; $136.3 ; 136.9 ; 138.4 ; 166.8 ; 177.1$. FT-IR $\left(\mathrm{cm}^{-1}\right) 1653$, 1717. Found: \%C 60.57, \%H 6.08, $\% \mathrm{~N}$ 4.65. $\mathrm{C}_{15} \mathrm{H}_{18} \mathrm{ClNO}_{3}$. Calculated: $\% \mathrm{C} 60.91, \% \mathrm{H} 6.13, \% \mathrm{~N} 4.74$. Unreacted anilide (124 mg, 59\%) was also isolated by flash chromatography (10:1 $\left.\mathrm{CH}_{2} \mathrm{Cl}_{2} / \mathrm{EtOAc}\right)$.<smiles>COC(=O)/C=C/c1ccc(OC)cc1NC(C)=O</smiles>

Methyl (E)-3-(2-acetylamino-4-methoxyphenyl)propenoate. Conditions: $1.5 \mathrm{hr}$ at $90{ }^{\circ} \mathrm{C}$. Flash chromatography $\left(4: 1 \mathrm{CH}_{2} \mathrm{Cl}_{2} / \mathrm{EtOAc}\right)$ afforded $212 \mathrm{mg}$ $(85 \%)$ of the product as a white solid, $\mathrm{mp} 169-170{ }^{\circ} \mathrm{C}$ (pentane); $\mathrm{R}_{\mathrm{f}}=0.15(5: 1$ $\mathrm{CH}_{2} \mathrm{Cl}_{2} /$ EtOAc); ${ }^{1} \mathrm{H}$ NMR $\left(\mathrm{CDCl}_{3}\right) \delta 2.16(\mathrm{~s}, 3 \mathrm{H}) ; 3.72(\mathrm{~s}, 3 \mathrm{H}) ; 3.74(\mathrm{~s}, 3 \mathrm{H}) ; 6.19(\mathrm{~d}, 1 \mathrm{H}$; $J=15.3 \mathrm{~Hz}, \mathrm{CH}=) ; 6.66(\mathrm{br} \mathrm{d}, 1 \mathrm{H} ; J=8.4 \mathrm{~Hz}) ; 7.21(\mathrm{~s}, 1 \mathrm{H}) ; 7.40(\mathrm{~d}, 1 \mathrm{H} ; J=8.4 \mathrm{~Hz})$; $7.70(\mathrm{~d}, 1 \mathrm{H} ; J=15.3 \mathrm{~Hz}, \mathrm{CH}=) ; 8.20($ br s, $1 \mathrm{H}) .{ }^{13} \mathrm{C} \mathrm{NMR}\left(\mathrm{CDCl}_{3}\right) \delta 23.9 ; 51.5 ; 55.3$; 
$109.8 ; 112.5 ; 116.5 ; 119.8 ; 127.9 ; 137.5 ; 139.3 ; 161.5 ; 167.6 ; 169.4 ;$ FT-IR $\left(\mathrm{cm}^{-1}\right) 1656$, 1711. Found: $\% \mathrm{C} 62.50, \% \mathrm{H} 6.07, \% \mathrm{~N}$ 5.46. $\mathrm{C}_{13} \mathrm{H}_{15} \mathrm{NO}_{4}$. Calculated: $\% \mathrm{C} 62.64, \% \mathrm{H}$ $6.07, \% \mathrm{~N} 5.62$.<smiles>COC(=O)/C=C/c1cc(Br)c(OC)cc1NC(=O)C(C)(C)C</smiles>

Methyl (E)-3-(5-bromo-4-methoxy-2-pivaloylaminophenyl)propenoate. Conditions: $3 \mathrm{hr}$ at $90{ }^{\circ} \mathrm{C}$. Analysis of the crude reaction mixture by $\mathrm{GC}$ revealed presence of the coupling product and unreacted anilide. No side products resulting from anilide were observed. Flash chromatography (20:1, then 10:1 $\left.\mathrm{CH}_{2} \mathrm{Cl}_{2} / \mathrm{EtOAc}\right)$ afforded $226 \mathrm{mg}(61 \%)$ of the product as a white solid: $\mathrm{mp} 189-190{ }^{\circ} \mathrm{C}$ $\left(\mathrm{CH}_{2} \mathrm{Cl}_{2}\right.$-pentane); $\mathrm{R}_{\mathrm{f}}=0.6\left(10: 1 \mathrm{CH}_{2} \mathrm{Cl}_{2} / \mathrm{EtOAc}\right) ;{ }^{1} \mathrm{H} \mathrm{NMR}\left(\mathrm{CDCl}_{3}\right) \delta 1.33(\mathrm{~s}, 9 \mathrm{H}) ; 3.74$ $(\mathrm{s}, 3 \mathrm{H}) ; 3.89(\mathrm{~s}, 3 \mathrm{H}) ; 6.24(\mathrm{~d}, 1 \mathrm{H} ; J=15.3 \mathrm{~Hz}, \mathrm{CH}=) ; 7.54(\mathrm{~s}, 1 \mathrm{H}) ; 7.58(\mathrm{~d}, 1 \mathrm{H} ; J=15.3$ $\mathrm{Hz}, \mathrm{CH}=) ; 7.65(\mathrm{~s}, 1 \mathrm{H}) .{ }^{13} \mathrm{C} \mathrm{NMR}\left(\mathrm{CDCl}_{3}\right) \delta 27.4 ; 39.9 ; 51.8 ; 56.4 ; 107.3 ; 107.9 ; 118.8$; $120.2 ; 131.2 ; 136.8 ; 137.4 ; 157.4 ; 167.0 ; 177.0$. FT-IR $\left(\mathrm{cm}^{-1}\right) 1652$, 1716. Found: \%C 52.11, \%H 5.45, \%N 3.62. $\mathrm{C}_{16} \mathrm{H}_{20} \mathrm{BrNO}_{4}$. Calculated: \%C 51.90, \%H 5.44, \%N 3.78.<smiles>COC(=O)/C=C/c1ccccc1N(C)C(C)=O</smiles>

Methyl (E)-3-(2-acetyl-N-methylaminophenyl)propenoate. A solution of $\mathrm{N}$-methylacetanilide $(300 \mathrm{mg}, 2.0 \mathrm{mmol})$ in DMF $(2 \mathrm{~mL})$ was added in 5 equal portions, during $1 \mathrm{hr}$, to the mixture consisting of methyl trans-3-bromopropenoate $(215 \mu \mathrm{L}, 2.0 \mathrm{mmol})$, silver triflate $(1.0 \mathrm{~mL}$ of a $2.5 \mathrm{M}$ solution, $2.5 \mathrm{mmol})$ and palladium (II) triflate $(0.6 \mathrm{~mL}$ of a $0.17 \mathrm{M}$ solution, $0.1 \mathrm{mmol})$. The solution of $\mathrm{Pd}(\mathrm{OTf})_{2}$ was prepared by mixing the solutions of $\mathrm{PdCl}_{2}$ and $\mathrm{AgOTf}$ in $\mathrm{DMF}$ (1/2 molar ratio). The mixture was heated for $2 \mathrm{hr}$ at $110{ }^{\circ} \mathrm{C}$ and worked up according to the general procedure. Flash chromatography $\left(5: 1 \mathrm{CH}_{2} \mathrm{Cl}_{2} /\right.$ EtOAc) afforded $185 \mathrm{mg}(40 \%)$ of the product as an oil; $\mathrm{R}_{\mathrm{f}}=0.3\left(5: 1 \mathrm{CH}_{2} \mathrm{Cl}_{2} / \mathrm{EtOAc}\right) ;{ }^{1} \mathrm{H} \mathrm{NMR}\left(\mathrm{CDCl}_{3}\right) \delta 1.75(\mathrm{~s}, 3 \mathrm{H}), 3.20(\mathrm{~s}, 3 \mathrm{H}) ; 3.79(\mathrm{~s}$, $3 \mathrm{H}) ; 6.44$ (d, $1 \mathrm{H} ; J=15.9 \mathrm{~Hz}, \mathrm{CH}=) ; 7.20$ (d, $1 \mathrm{H} ; J=7.8 \mathrm{~Hz}) ; 7.42(\mathrm{~m}, 2 \mathrm{H}) ; 7.64(\mathrm{~d}, 1 \mathrm{H}$; $J=15.9 \mathrm{~Hz}, \mathrm{CH}=) ; 7.69(\mathrm{dd}, 1 \mathrm{H} ; J=1.5 \mathrm{~Hz}$ and $7.8 \mathrm{~Hz}) .{ }^{13} \mathrm{C} \mathrm{NMR}\left(\mathrm{CDCl}_{3}\right) \delta 22.3,36.9$; $51.9,121.1 ; 127.7 ; 128.7 ; 128.8 ; 131.6 ; 132.1 ; 138.5 ; 143.5 ; 166.6 ; 170.6$. FT-IR $\left(\mathrm{cm}^{-1}\right)$ 1650, 1699. Found: $\% \mathrm{C} 67.00, \% \mathrm{H} 6.68, \% \mathrm{~N}$ 5.84. $\mathrm{C}_{13} \mathrm{H}_{15} \mathrm{NO}_{3}$. Calculated: $\% \mathrm{C} 66.94$, $\% \mathrm{H} 6.48, \% \mathrm{~N} 6.00$. 
<smiles>COC(=O)/C=C/c1ccc(O)cc1NC(=O)C(C)(C)C</smiles>

Methyl (E)-3-(4-hydroxy-2-pivaloylaminophenyl)propenoate. Conditions: $1 \mathrm{hr}$ at $90{ }^{0} \mathrm{C}$. Dilution of the reaction mixture with toluene $(50$ $\mathrm{mL})$, filtration, washing of organic solution with water $(2 \times 10 \mathrm{~mL})$, filtration through a short pad of silica gel $\left(\mathrm{CH}_{2} \mathrm{Cl}_{2}\right)$ and evaporation of the solvent afforded $179 \mathrm{mg}(65 \%)$ of the product as a brownish solid. $\mathrm{Mp} 157-158{ }^{\circ} \mathrm{C}$ (pentane, colorless crystals); $\mathrm{R}_{\mathrm{f}}=0.2$ $\left(10: 1 \mathrm{CH}_{2} \mathrm{Cl}_{2} / \mathrm{EtOAc}\right) ;{ }^{1} \mathrm{H}$ NMR $\left(\mathrm{CDCl}_{3}\right) \delta 1.32(\mathrm{~s}, 9 \mathrm{H}) ; 3.72(\mathrm{~s}, 3 \mathrm{H}) ; 6.22(\mathrm{~d}, 1 \mathrm{H} ; J=$ $15.6 \mathrm{~Hz}, \mathrm{CH}=) ; 6.64(\mathrm{dd}, 1 \mathrm{H} ; J=2.4$ and $8.4 \mathrm{~Hz}) ; 7.38(\mathrm{~s}, 1 \mathrm{H}) ; 7.38(\mathrm{~d}, 1 \mathrm{H} ; J=8.4 \mathrm{~Hz})$; $7.68(\mathrm{~d}, 1 \mathrm{H} ; J=15.6 \mathrm{~Hz}, \mathrm{CH}=) ; 7.81(\mathrm{~s}, 1 \mathrm{H}) ; 9.00(\mathrm{~s}, 1 \mathrm{H}) .{ }^{13} \mathrm{C} \mathrm{NMR}\left(\mathrm{CDCl}_{3}\right) \delta 27.4$; $39.9 ; 51.7 ; 111.6 ; 114.3 ; 116.7 ; 118.2 ; 128.7 ; 136.6 ; 139.1 ; 159.7 ; 167.7 ; 178.6$. FT-IR $\left(\mathrm{cm}^{-1}\right)$ 1649, 1701, 3300 (br). Found: $\% \mathrm{C}$ 64.82, \%H 6.87, \%N 4.88. $\mathrm{C}_{15} \mathrm{H}_{19} \mathrm{NO}_{4}$. Calculated: \%C 64.97, \%H 6.91, \%N 5.05.<smiles>COC(=O)/C=C/c1ccc(OC)cc1N(C)C(C)=O</smiles>

Methyl (E)-3-(4-methoxy-2-acetyl-N-methylaminophenyl)propenoate. Conditions: $1.5 \mathrm{hr}$ at $90{ }^{\circ} \mathrm{C}$. Dilution of the reaction mixture with $\mathrm{CH}_{2} \mathrm{Cl}_{2}$, filtration through a short pad of silica gel and evaporation of the solvent afforded $257 \mathrm{mg}$ (98\%) of the product as a yellowish solid. $\mathrm{Mp} 127-128{ }^{\circ} \mathrm{C}\left(\mathrm{CDCl}_{3}\right.$-pentane, colorless needles); $\mathrm{R}_{\mathrm{f}}=0.45\left(5: 1 \mathrm{CH}_{2} \mathrm{Cl}_{2} /\right.$ EtOAc); ${ }^{1} \mathrm{H}$ NMR $\left(\mathrm{CDCl}_{3}\right) \delta 1.75(\mathrm{~s}, 3 \mathrm{H}) ; 3.17(\mathrm{~s}, 3 \mathrm{H})$; $3.73(\mathrm{~s}, 3 \mathrm{H}) ; 3.78(\mathrm{~s}, 3 \mathrm{H}) ; 6.30(\mathrm{~d}, 1 \mathrm{H} ; J=16.2 \mathrm{~Hz}, \mathrm{CH}=) ; 6.70(\mathrm{~d}, 1 \mathrm{H} ; J=2.4 \mathrm{~Hz}) ; 6.90$ $(\mathrm{dd}, 1 \mathrm{H} ; J=2.4$ and $8.7 \mathrm{~Hz}) ; 7.53(\mathrm{~d}, 1 \mathrm{H} ; J=16.2 \mathrm{~Hz}, \mathrm{CH}=) ; 7.61(\mathrm{~d}, 1 \mathrm{H} ; J=8.7 \mathrm{~Hz}$,). ${ }^{13} \mathrm{C}$ NMR $\left(\mathrm{CDCl}_{3}\right) \delta 22.1 ; 36.8 ; 51.2 ; 55.6 ; 113.5 ; 114.8 ; 118.4 ; 124.2 ; 128.9 ; 138.1$; $144.7 ; 162.1 ; 166.9 ; 170.7$; FT-IR $\left(\mathrm{cm}^{-1}\right) 1669,1717$. Found: $\% \mathrm{C} 63.83, \% \mathrm{H} 6.41, \% \mathrm{~N}$ 5.22. $\mathrm{C}_{14} \mathrm{H}_{17} \mathrm{NO}_{4}$. Calculated: \% $63.87, \% \mathrm{H}$ 6.51, \%N 5.32.<smiles>COc1ccc(/C=C/C(=O)c2ccccc2)c(N(C)C(C)=O)c1</smiles>

(E)-3-(4-methoxy-2-acetyl-N-methylaminophenyl)-1-

phenylpropenone. The reaction was performed according to general procedure on a 0.5 mmol scale using cis-1-phenyl-3-bromo-2-propenone $(110 \mathrm{mg}, 0.52 \mathrm{mmol})$ as the olefin component and $10 \mathrm{~mol}^{\circ} \mathrm{PdCl}_{2}(0.2 \mathrm{~mL}$ of a $0.25 \mathrm{M}$ solution in DMF, $0.05 \mathrm{mmol})$. Conditions: $3 \mathrm{~h}$ at $90{ }^{\circ} \mathrm{C}$. After purification by flash chromatography $(5: 1$ $\mathrm{CH}_{2} \mathrm{Cl}_{2} /$ EtOAc), $133 \mathrm{mg}(43 \%)$ of product was obtained as a brownish solid, $\mathrm{mp} 135-$ $136{ }^{0} \mathrm{C}\left(\mathrm{CH}_{2} \mathrm{Cl}_{2}\right.$-pentane $) ; \mathrm{R}_{\mathrm{f}}=0.4\left(5: 1 \mathrm{CH}_{2} \mathrm{Cl}_{2} / \mathrm{EtOAc}\right) ;{ }^{1} \mathrm{H} \mathrm{NMR}\left(\mathrm{CDCl}_{3}\right) \delta 1.81(\mathrm{~s}, 3 \mathrm{H})$; $3.23(\mathrm{~s}, 3 \mathrm{H}) ; 3.86(\mathrm{~s}, 3 \mathrm{H}) ; 6.74(\mathrm{~d}, 1 \mathrm{H} ; J=2.1 \mathrm{~Hz}) ; 6.97(\mathrm{dd}, 1 \mathrm{H} ; J=2.1$ and $8.7 \mathrm{~Hz}) ; 7.43$ $(\mathrm{d}, 1 \mathrm{H} ; J=15.3 \mathrm{~Hz}) ; 7.52(\mathrm{~m}, 2 \mathrm{H}) ; 7.73(\mathrm{~d}, 1 \mathrm{H} ; J=15.3 \mathrm{~Hz}, \mathrm{CH}=) ; 7.77(\mathrm{~d}, 1 \mathrm{H} ; J=8.7$ 
$\mathrm{Hz}) ; 7.99(\mathrm{~d}, 1 \mathrm{H} ; J=7.5 \mathrm{~Hz}) .{ }^{13} \mathrm{C}$ NMR $\left(\mathrm{CDCl}_{3}\right) \delta 22.2 ; 35.9 ; 55.6 ; 113.9 ; 114.7 ; 122.3$; $124.9 ; 128.4 ; 128.6 ; 129.9 ; 132.9 ; 137.9 ; 138.5 ; 145.4 ; 162.2 ; 170.4 ; 189.9 ; 195.0$. FT-IR $\left(\mathrm{cm}^{-1}\right)$ 1658. Found: \%C 73.38, \%H 6.12, \%N 4.39. $\mathrm{C}_{19} \mathrm{H}_{19} \mathrm{NO}_{3}$. Calculated: \%C 73.77, $\% \mathrm{H} 6.19, \% \mathrm{~N} 4.53$.<smiles>[2H]c1cc(C)ccc1NC(C)=O</smiles>

2-Deuterio-4-methylacetanilide. To a stirred solution of 2-bromo-N(BOC)-4methylaniline $^{11}(1.65 \mathrm{~g}, 6.45 \mathrm{mmol})$ in dry THF $(50 \mathrm{~mL}) \mathrm{MeLi}$ (about $5 \mathrm{~mL}$ of a $1.6 \mathrm{M}$ solution in ether) was added until persistent yellow color developed. The reaction mixture was cooled to $-40^{\circ} \mathrm{C}$ and $1.7 \mathrm{M} \mathrm{t}-\mathrm{BuLi}$ in pentane $(7.0 \mathrm{~mL}, 11.9 \mathrm{mmol})$ was added in about 10 min. $^{13}$ Then $\mathrm{D}_{2} \mathrm{O}(0.7 \mathrm{~mL})$ was added and the reaction mixture was allowed to warm to room temperature, solvent was evaporated and residue extracted with hexanes $(50 \mathrm{~mL})$. The extract was filtered and solvent was evaporated to yield $1.25 \mathrm{~g}$ of a yellow solid. Recrystallization of sample from pentane gave 2,N-dideuterio-4-methyl-N(BOC)acetamide as long white needles. ${ }^{1} \mathrm{H}$ NMR $\left(\mathrm{CDCl}_{3}\right) \delta 1.51(\mathrm{~s}, 9 \mathrm{H}) ; 2.29(\mathrm{~s}, 3 \mathrm{H}) ; 7.08(\mathrm{~m}$, $2 \mathrm{H}$ ); 7.22 (br d, $1 \mathrm{H} ; J=8.7 \mathrm{~Hz}$ ). The Boc group was hydrolyzed in the following way. A $1 \mathrm{M}$ solution of $\mathrm{HCl}$ in $\mathrm{MeOH}$ was prepared by addition of $\mathrm{AcCl}(1.0 \mathrm{~mL})$ to cold $\mathrm{MeOH}$ $(12 \mathrm{~mL})$. The Boc-protected aniline was dissolved in this mixture and the solution kept at RT for $2 \mathrm{hr}$. Neutralization of the solution with $\mathrm{Na}_{2} \mathrm{CO}_{3}(5 \%$ aq. solution), extraction with dichloromethane $(3 \times 15 \mathrm{~mL})$ and evaporation of the solvent gave 2-deuterio-4methylaniline as a brownish solid. ${ }^{1} \mathrm{H}$ NMR $\left(\mathrm{CDCl}_{3}\right) \delta 2.24(\mathrm{~s}, 3 \mathrm{H}) ; 3.52(\mathrm{br} \mathrm{s}, 1 \mathrm{H}) ; 6.61$ $(\mathrm{d}, 1 \mathrm{H} ; J=7.8 \mathrm{~Hz}) ; 6.97(\mathrm{~m}, 2 \mathrm{H})$. ). Crude aniline was dissolved in $\mathrm{Ac}_{2} \mathrm{O}(3 \mathrm{~mL})$ and $\mathrm{kept}$ at RT for $15 \mathrm{~min}$. Evaporation of the solvent in vacuo afforded 2-deuterio-4methylacetanilide as a yellowish solid $(0.92 \mathrm{~g}, 96 \%, 98 \%$ isotopic purity, based on relative intensity of $\mathrm{M}^{+\cdot}=150$ and $\mathrm{M}^{+\cdot}=149$ in $\left.\mathrm{MS}\right)$. Recrystallization from water afforded colorless crystals. ${ }^{1} \mathrm{H}$ NMR $\left(\mathrm{CDCl}_{3}\right) \delta 2.14(\mathrm{~s}, 3 \mathrm{H}) ; 2.30(\mathrm{~s}, 3 \mathrm{H}) ; 7.10(\mathrm{~m}, 2 \mathrm{H})$; 7.36 (m, 1H; $J=8.7 \mathrm{~Hz}$ ); 7.50 (br s, 1H); MS, m/z, (\%): 151(3.2), 150(35.1), 149(0.9), 108(90.5), 107(100.0).<smiles>[2H]c1cc(C)cc([2H])c1NC(C)=O</smiles>

2,6-Dideuterio-4-methylacetanilide. A solution of 4-methylacetanilide $(0.54 \mathrm{~g}, 0.5 \mathrm{mmol})$ in $35 \% \mathrm{DCl} / \mathrm{D}_{2} \mathrm{O}(5 \mathrm{~mL})$ was heated for $12 \mathrm{~h}$ at $100{ }^{0} \mathrm{C}$ in a Schlenk storage/reaction vessel (100 mL capacity). CAUTION: load flask to $10 \%$ capacity only! High pressure may develop. Solvent was evaporated in vacuo. Procedure was repeated 3 times. The residue was dissolved in $28-30 \%$ ammonia $(10 \mathrm{~mL})$ and left for $2 \mathrm{~h}$ at $\mathrm{rt}$ to exchange N-D for N-H. Extraction with $\mathrm{CH}_{2} \mathrm{Cl}_{2}(3 \times 5 \mathrm{~mL})$, evaporation of the solvent followed by acetylation $\left(1: 1 \mathrm{Ac}_{2} \mathrm{O}-\mathrm{CH}_{2} \mathrm{Cl}_{2}, \mathrm{rt}, 15 \mathrm{~min}\right)$, and concentration in vacuo 
provided 2,6-dideuterio-4-methylacetanilide as a white solid in 93\% yield and $98 \%$ isotopic purity (according to mass spectroscopy). ${ }^{1} \mathrm{H}$ NMR $\left(\mathrm{CDCl}_{3}\right) \delta 2.16$ (s, 3H); 2.31 (s, 3H); 7.13 (s, 2H), 7.45 (br s, 1H).<smiles>[2H]c1cc(C)cc(/C=C/C(=O)OC)c1NC(C)=O</smiles>

\section{intermolecular competition reactions.}

Determination of kinetic isotope effects by intra- or

A. Intramolecular competition reaction. A solution of 2-deuterio-4methylacetanilide $(75 \mathrm{mg}, 0.5 \mathrm{mmol})$ in DMF $(1 \mathrm{~mL})$ was mixed with a solution of silver triflate in DMF $(0.25 \mathrm{~mL}$ of a $2.5 \mathrm{M}$ solution, $0.63 \mathrm{mmol})$, a solution of palladium chloride in DMF $(0.1 \mathrm{~mL}$ of a $0.25 \mathrm{M}$ solution, $0.025 \mathrm{mmol})$, and methyl trans-3-bromo2-propenoate $(0.055 \mathrm{~mL}, 0.5 \mathrm{mmol})$. The mixture was kept in a screw-cap vial at $70{ }^{\circ} \mathrm{C}$ for $2.5 \mathrm{hr}$ until approximately $50 \%$ conversion to coupling product was observed by GC. The reaction mixture was worked up as described in general procedure. Ratio of the deuterated/non-deuterated product 2-D/2 was found to be $3.6: 1$ by ${ }^{1} \mathrm{H}$ NMR (integration of a doublet at $\delta 7.55$ for non-deuterated 2 relative to a doublet at $\delta 7.77$ for $\mathrm{CH}=$ for both 2 and 2-D). The isotope effect $\mathrm{k}_{\mathrm{H}} / \mathrm{k}_{\mathrm{D}}=3.6 / 1$. Unreacted starting material was recovered and was found to have ${ }^{1} \mathrm{H}$ NMR and MS spectra identical to that of 2deuterio-4-methylacetanilide indicating no $\mathrm{H}-\mathrm{D}$ scrambling in the reaction.

B. Intermolecular competition reaction. Reaction was performed according to the general procedure using 1:1 mixture of 4-methylacetanilide $(75 \mathrm{mg})$ and 2,6-dideuterio-4methylacetanilide $\left(75 \mathrm{mg}\right.$ ). After $1 \mathrm{hr}$ at $90{ }^{\circ} \mathrm{C} 36 \%$ conversion to the product (GC data) was observed. Product was separated from the starting material by column chromatography $\left(\mathrm{CH}_{2} \mathrm{Cl}_{2}\right.$ followed by $\left.\mathrm{CH}_{2} \mathrm{Cl}_{2} / \mathrm{EtOAc} 10 / 1\right)$. In the product 2/2-D ratio was found to be 2.05: 1 (NMR integration of a doublet at $\delta 7.55$ for non-deuterated 2 relative to a doublet at $\delta 7.78$ for both $\mathbf{2}$ and 2-D). Residual starting material was shown to consist of non-deuterated and dideuterated anilide (1/1.77 ratio). This was determined by ${ }^{1} \mathrm{H}$ NMR integration of a doublet at $\delta 7.38$ for non-deuterated vs. a multiplet at $\delta 7.13$ for both non- and dideuterated 4-methylacetanilides. No significant deuterium scrambling in recovered anilides was observed by mass spectroscopy. Mathematical treatment according to Ismagilov ${ }^{14}$ gave $\mathrm{k}_{\mathrm{H}} / \mathrm{k}_{\mathrm{D}}=3.8$ based on unreacted anilide and $\mathrm{k}_{\mathrm{H}} / \mathrm{k}_{\mathrm{D}}=3.6$ based on the product. Ismagilov's treatment of kinetic resolutions is applicable here due to identical kinetic scheme.

Product inhibition. Two parallel reactions were set up.

A. The reaction of 4-methylacetanilide with bromoester was run according to the general procedure on a $0.1 \mathrm{mmol}$ scale. After $1.5 \mathrm{hr}$ at $90{ }^{\circ} \mathrm{C} 51 \%$ conversion to the product 2 was observed by GC.

B. The reaction was performed as above with the addition of coupling product 2 (23 $\mathrm{mg}, 0.1 \mathrm{mmol}$ ). After $1.5 \mathrm{hr}$ at $90{ }^{0} \mathrm{C} 16 \%$ conversion to the product 2 was observed by GC. No change in conversion was observed after $40 \mathrm{~min}$. 


\section{References}

1. Kurtz A. N., Billups W. E., Greenlee R. B., Hamil H. F., Pace W. T. J. Org. Chem. 1965, 30, 3141 .

2. Weir, J. R.; Patel, B. A.; Heck, R. F. J. Org. Chem. 1980, 45, 4926.

3. Piers, E.; Wong, T.; Coish, P. D.; Rogers, C. Can. J. Chem. 1994, 72, 1816.

4. Ma, S.; Lu, X.; Li, Z. J. Org. Chem. 1992, 57, 709.

5. Fuhrer, W.; Gschwend, H. W. J. Org. Chem. 1979, 44, 1133.

6. Katritzky, A. R.; Eynde, V.; Jacques, J. J. Chem. Soc., Perkin Trans. 1, 1989, 639.

7. Sveshnikov, N. N.; Levkoev, I. I. Zh. Obshch. Khim. 1946, 16, 1071.

8. Gannett, P. M.; Johnson II, E. M.; Grimes, M. A.; Myers, A. L.; Deavers III, R. E.; Tracy, T. S. J. Labelled Compd. Rad. 2003, 46, 107.

9. Hillis, L. R.; Gould, S. J. J. Org. Chem. 1985, 50, 718.

10. Yamasaki R., Tanatani, A.; Azumaya, I.; Saito, S.; Yamaguchi, K.; Kagechika, H. Org. Lett. 2003, 5, 1265.

11. Kessler, A.; Coleman, C. M.; Charoenying, P.; O'Shea, D. F. J. Org. Chem. 2004, 69, 7836.

12. Ripka, W. C.; Applequist, D. E. J. Am. Chem. Soc. 1967, 89, 4035.

13. For a related lithiation procedure, see: Wender, P. A.; White, A. W. Tetrahedron 1983, 39, 3767.

14. Ismagilov, R. F. J. Org. Chem. 1998, 63, 3772. 